\title{
From Control to Coordination: Mahalanobis Distance - Pattern Approach to Strategic Decision Making
}

\author{
Shuichi FUKUDA \\ Keio University, Systems Design and Management, Japan
}

\begin{abstract}
Up to now, our engineering has been control-based. The goal was clear, We made efforts to get to the goal faster and more effectively. This was possible because changes were smooth, so we could differentiate them mathematically. Therefore, we could predict the future. But today, changes become sharp and unpredictable. Therefore, adaptability becomes more important than efficiency. Or to describe it another way, we need to find an appropriate goal first by trial and error. Yesterday tactics was important, but today strategy becomes important. But our world becomes so complex and complicated that we need to work as a team. And the formation of this team must vary from situation to situation to win the game. Therefore, we must develop a truly adaptive network. This paper proposes that if we introduce non-Euclidean Mahalanobis Distance (MD) and combine it with patterns, then we can develop a holistic and quantitative performance indicator. It evaluates our performance in a very short time for almost any number of dimensions, because it is free from the constraints of orthonormality and units in Euclidean Space. Thus, this Mahalanobis Distance-Pattern (MDP) Approach helps us to make an approriate strategic decision.
\end{abstract}

Keywords. Strategic Decision Making, Unpredictable Changes, Control, Coordination, Management, How to Win the Game, Self-Decision, Psychological Satisfaction and Happiness

\section{Introduction}

This paper points out how the real world is changing. Although there were changes yesterday, they changed smoothly, so we could predict the future. Thus, the idea of Control played a leading role. Therefore, we evaluated product value and tactics was important. Reproducibility and Rationality were key ideas. But today, the real world changes frequently and extensively and what makes it worse, in an unpredicable manner. Thus, the word Adaptability is attracting wide attention. But if we wish to make our dreams come true, we need to outsmart the environments and situations.

To solve this problem, we need to work together with machines (robots) on the same team, as IoT indicates. To work as a human-machine team to cope flexibily and adaptively with drastic changes, we need to be in the system. We used to give instruction to muchines from outside and machines followed. But to effectively and adaptively respond to the changes, we need a communication tool and we need a performance indicator to know how well we are doing. As to communication, Movements are a common basis for machines and humans. But current researches on 
movements are based on the idea of Control. When the environments and situations change rapidly, we need Coordination. In fact, humans coordinate many body parts and balance our bodies. In short, our world is changing from Tactics to Strategy and what and how decisions should be done become crucially important.

This paper proposes Mahalanobis Distance Pattern (MDP) Approach for supporting Strategic Decision Making and as the number of degrees of freedom becomes tremendously large, we leave the decision to our Instinct, because the direct interaction with the real world becomes indispensable and to avoid computational complexity, we can utilize our Instinct as the octopus is doing. MDP helps us to perceive the real world and provide the holistic and quantitative performance indicator to move in the adequate way and in our own personal way.

The main core of this paper is since the Industrial Revolution, we have been working for others. i.e., for external rewards, but in the next generation engineering, working for your own Self becomes crucially important. In short, our engineering will change from material satisfaction to mental satisfaction. $M D P$ will enable us to enjoy mental satisfaction and happiness.

\section{From Control to Coordination}

Importance of Coordination is rapidly increasing. Up to now, engineering has focused on Control. This is because changes yesterday were smooth, so we could differentiate them mathematically. In other words, we could predict the future. Thus, engineers could foretell the operating conditions of their products. Therefore, their focus has been on products and on how they could control them.

But today the changes are sharp. Therefore, we cannot predict the future. Engineers cannot estimate the operating conditions of their products anymore (Figure 1).

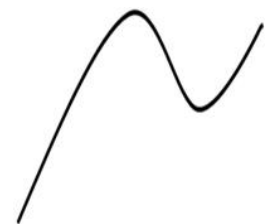

Yesterday

Smooth Change

Differentiable

Predictable

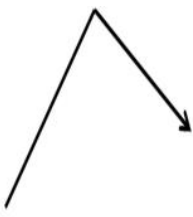

Today

Sharp Change

Not Differentiable

Not Predictable

Figure 1. Changes of yesterday and today.

And our world keeps on expanding rapidly and changed from a small, closed world with boundaries to a wide, open world without boundaries. Such changes make it very difficult to apply rational approaches. Therefore, Coordination or Strategic Management is increasing its imporance. But we do not have good tools for esttimating our performance.

Why we have difficulty in making adequate decisions to cope with the changing situations is because we have been stuck with Euclidean Space approaches. If we introduce Non-Euclidean Space approach, we can free ourselves from the constraints of 
orthonormality and units and expand dimensions easily. Mahalanobis Distance (MD) is one of such Non-Euclidean tools.

This paper points out that if we introduce $M D$ and combine it with Patterns, we can evaluate our Strategic Decision Making performance holistically and quantitatively.

\section{The Real World is Changing from Predictable to Unpredictable}

When our world was closed with boundaries, we could apply rational approaches in a straightforward way. But as our world changed to wide, open without boundaries and the changes become unpredictable, we cannot apply rational approaches anymore.

\section{System Identification Approach: \\ Expanding Rational World to Controllable World}

To cope with this problem, engineers invented System Identification Approach, Its basic idea is the same as the one we use to indentify the name of a river. If we look at the river (flow) itself, we cannot identify its name, because it changes every minute. But if we look around, we can find mountains or forests that do not change. So, we can identify its name (Figure 2).

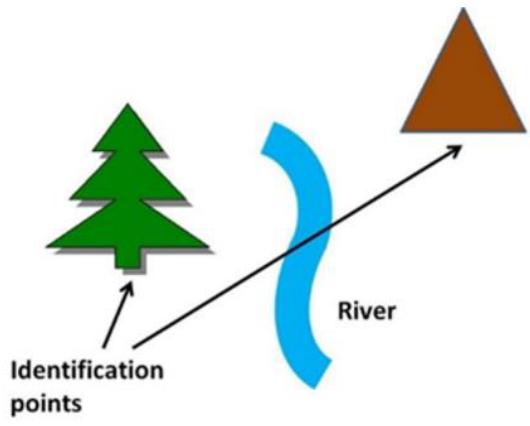

Figure 2. Identifying the name of a river.

System Identification Approach pays attention to such feature points to identify the system. Once the system is identified, we can identify its parameters. Thus, we succeeded in expanding the Rational World and established the Controllable World.

But as changes take place more and more frequently and extensively, and in an unpredictable manner, System Identification Approach does not work anymore.

In short, we have been discussing on the bank of a river, but we are now thrown into the river. So, we need to swim against the flow to get to the goal we have on our mind. Strictly speaking, the word Adaptability is not appropriate. If we only adapt to the flow, we will be carried away and we cannot get to our goal. We need to overcome the flow to get to the goal. It is nothing else but a game and we need to win the game against the enemy, i.e., the changing real world.

Then, how can we overcome the environment and situation and get to where we want to? As Movement is essential in product performance, let us take up here Human Movement to understand why Coordination becomes more important than Control. 


\section{Human Movement}

Nikolai Berstein, famous human motion control researcher, showed us how Human Movement trajectories vary widely [1],[2], (Figure 3).

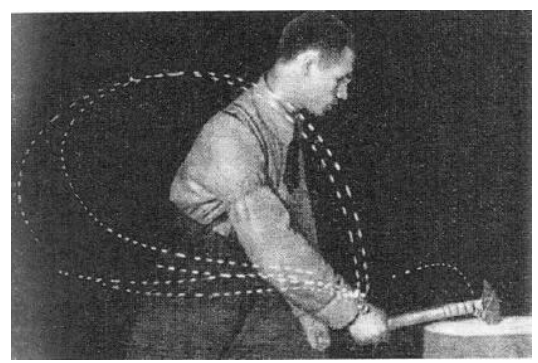

Figure 3. Cyclogram of hammering.

But such variations are observed only at the early stage. When we get close to the target object, our trajectories are fixed. Almost all motion studies or robotics deal with this stage. At this stage, the number of degrees of freedom is reduced to the minimum and as the trajectories are fixed, we can apply rational approaches. In other words, we can Control them.

Then, why do our trajectories vary so widely from situation to situation at the early stage? It is because we, humans, Coordinate many body parts and Balance our body.

There are two kinds of Humna Movements. Motion is movement we observe from the outside. Motor is within ourselves. Motion is made clear as Bernstein showed us using his cyclogram. But as to Motor, almost all is still unclear, although particular research is being carried out, We do not know anything about it as a whole.

Bernstein pointed out that the problem of tremendously large number of degrees of freedom makes the study of Human Movement very difficult and that it is not Control, but Coordination which we should consider to study Human Movement.

In fact, at the early stage when our trajectories vary widely, many different Muslces are mobilized. Which Muscles are mobilized varies from case to case. But when we get close to the target obect, Muscles harden and we do not need to consider Muscle Movements at all. They move together with our Skeleton. Thus, almost all Motion Control studies are based on Skeleton Movements. They do not consider Muscle Movements. But at the early stage, Muscles work from one muscle to another. Many different Muscles work in response to the changing situation. We, humans, use many different parts of our body to respond to the changing environment and situation.

\section{Increasing Importance of Strategic Decision Making: Five Sectors of the Economy}

Interestingly enoough, economists are proposing the quinary sector of the economy. The primary sector is agriculture, fishing, hunting, etc. The secondary sector is manufaturing, etc. The tertial sector is service industry. The quaternary sector is ICT. These are well-known. 
The quinary sector is Decision Making. Economists point out that what tools we use and what pieces of information we use become increasingly important. Thus, they insist Decision Making will be the next sector of the economy (Table 1).

Table 1. Five sectors of the economy.

\begin{tabular}{|l|l|}
\hline Sector & Activities \\
\hline 5 Quinary Sector & Decision Making \\
\hline 4 Quaternary Sector & Knowledge and ICT Industry \\
\hline 3 Tertiary Sector & Service Industry \\
\hline 2 Secondary Sector & Transforms raw materials into Products - Manufacturing, etc. \\
\hline 1 Primary Sector & Extracts raw materials from nature - Agriculture, Fishing, etc. \\
\hline
\end{tabular}

\section{Team Formation Cooresponding to the Changing Situation}

Then, how can we make right decisions in engineering today, when environments and situations change frequently, extensively and in an unpredictable manner?

As three heads are better than one, we need a team to cope with this problem. And this team should be a network, because all nodes in a network can be an output node so that flexible response can be secured. But we need more than that. We need a network that changes flexibly with the changes of the real world.

If we take up soccer, I think we can understand easily. Its formation yesterday did not change. All players are expected to do his best at his own position, because the game did not change much. But today, the game changes frequently and extensively and you cannot predict what strategy your enemy will take. So, you must change your formation very flexibly and adaptively to win the game.

Managers were outside of the pitch yesterday, but today they are on the pitch. In most cases, midfielders are playing-managers. And other players must be prepared proactively to respond to his instructions. The formation changes drastically. It may be 3 player or 4 player formation, depending on the situation and other playes must be prepared in advance to form such game-winning formation. Thus, Coordination becomes crucial in soccer. Which players to use or what formation to take. We must make decisions.

\section{How Can We Coordinate? It Requires Trials and Errors.}

But such Coordination is carried out by trial and error. In short, Coordinaion requires Pragmatic Approach. Learning from Failures is emphasized in Pragmatism. But there is no appropriate tool to assist us with the task how to practice it. This is because appropriate performance indicators are lacking.

In engineering, we use Euclidean Space tools in most cases. But Euclidean Space reequires orthonormality and units. So with increasing dimensionality, Euclidean Space approach becomes increasingly difficult to apply.

\section{Mahalanobis Distance (MD)}

But if we introduce Non-Euclidean Space tool, then we can free ourselves from the constraints of orthonormality and units. Mahalanobis Distance is originally proposed to 
identify the outliers. It indicates how much the point $\mathrm{P}$ is far away from the mean of the dataset. Note that this dataset can be any dataset. It does not have to be normally distributed (Figure 4).

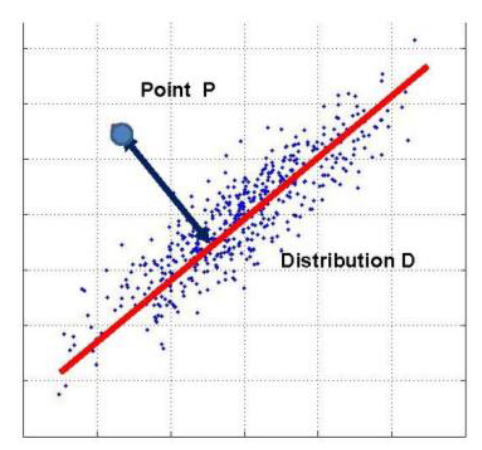

Figure 4. Mahalanobis Distance (MD).

Mahalanbis Distance (MD) is defined as

$\mathrm{MD}=($ Standard deviation $) /($ mean $)$

So, $M D$ is not a distance in the sense of Euclidean Space. It is a quotient.

And each $M D$ is independent. No orthogonality is required. So, we can increase dimensionality easily.

But to understand the whole picture, we need to know how each $M D$ is related. Introduction of Patterns enables us to do such a holistic performance evaluation. Thus, if we combine $M D$ and Patterns, we can develop a holistic and quantitative performance indicator.

\section{Patterns: Its Advantage}

Fukuda and his group used to study how we can extract emotion from face. They tried many image processing technques, but these techniques took too much time and without any satisfying results.

While repeating such trials, Fukuda realized we can extract emotion from the cartoon face without any difficulty. At that time, cartoons were in black and white and were very simple. Still, we could understand the emotion of characters at once.

So, his group developed cartoon face model and instead of processing images, they compared the real face with the cartoon face model and extracted emotion from face very easily [4], [5], (Figure 5). 


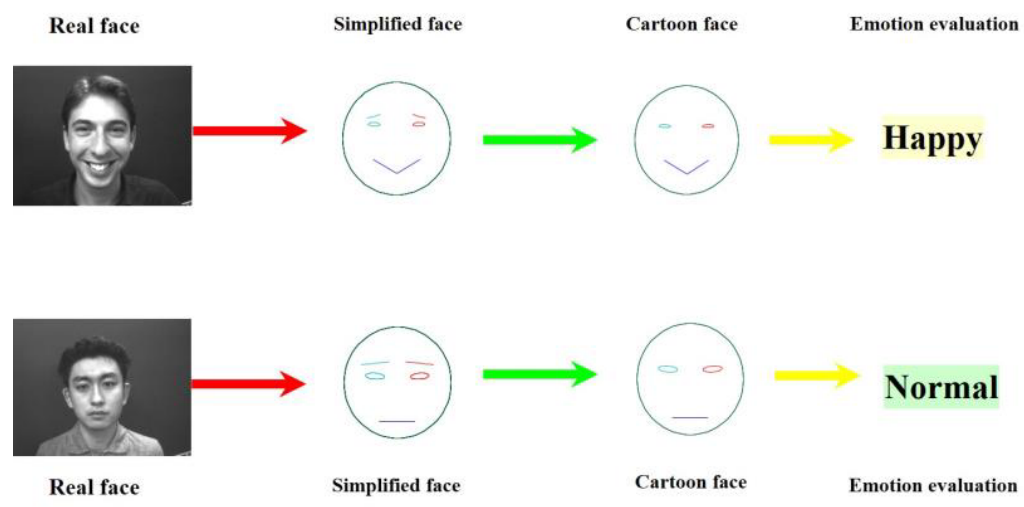

Figure 5. Cartoon Face Model.

In this study, comparison between the real face and the cartoon face was carried out, based on Euclidean Space approach. But the number of dimensions is very small, so it did not produce any problem at all.

But when the dimensions come to be very large, such Euclidean Space approach does not work effectively. But if we introduce $M D$ and combine it with Patterns, then we can understand easily how better or worse we are performing.

\section{Mahalanobis Taguchi System (MTS)}

Genichi Taguchi developed Mahalanobis Taguchi System (MTS) [6] about the same time. We should note that MTS is completely different from well-known Taguchi Method (TM). TM controls quality element by element or part by part and it is based on Eulidean Space approach. But MTS is factory-based holistic Quality Management. It is not Control, but Coordination. Different sectors of a factory work collaboratively to achieve the desired quality of a final product.

Taguchi realized that if he introduces $M D$ to quality management, such factories which cannot control quality part by part can manage quality as a factory. $M D$ is free from orthonormality and units, so they can apply easily. But they cannot evaluate how good they are doing as a whole. Taguchi realized that if he combines MD with Patterns, then they can see the whole picture.

MTS goes in the following way. Let us take number example here. Number 2 is written in many different ways from person to person. But if we average those which can be identified as number 2, we can get a standardized number 2 (Figure 6). We call this standardarized number 2 Unit Space.

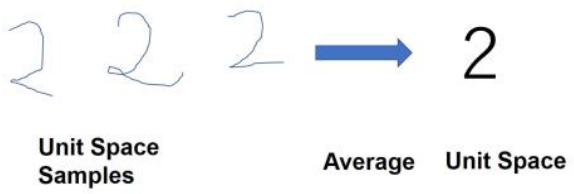

Figure 6. Defining Unit Space. 
Then we set the allowable threshold (Figure 7), If $M D$ is smaller than this threshold, the number can be identified as number 2. If $M D$ is larger than the threshold, we cannot regard it as number 2 .

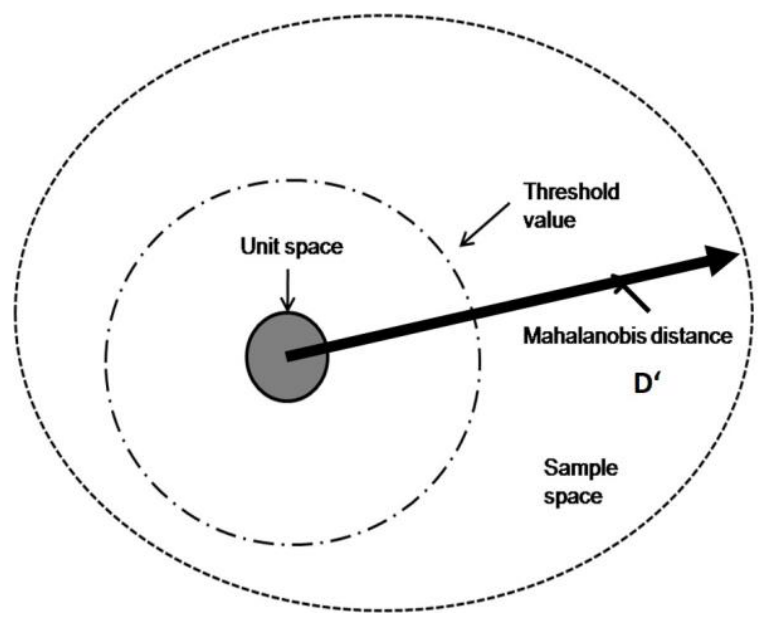

Figure 7. Mahalanobis Taguchi System (MTS).

We should note that images can be processed in the same way, because images are represented as pixel sequence (Figure 8). So, it boils down to patterns.

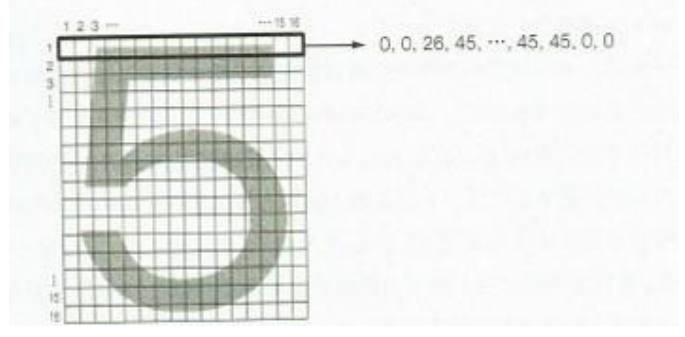

Figure 8. Pixels to pattern.

MTS is welcomed by many companies. These companies cannot carry out part by part quality control. But they can manage quality holistically or on a factory basis.

\section{Mahalanobis Distance-Pattern (MDP) Approach}

The merit of MTS is factories can perform Strategic Decision Making. Other quality tools are control-based or tactics-based. Its merit is the goal is clear from the first, so factroies can make efforts to reduce time and cost.

But today environments and situations change frequently and extensively, and in an unpredicable manner so it becomes more and more diffiulct for factories to identify what element or part to control. Many different sectors share the same problem. So, it 
is pointed out widely that Adaptability is becoming more important than Control. But Adaptability is not enough. What we should pursue is to overcome the situation.

Thus, MTS is very useful, because industries do not have to find paratmeters to control. This is why System Identification Approach is no more effective.

MDP Approach measures "how performance changes with each movement". The decision is left to humans, or to be exact, it is Instinct. Utilizing Instinct, we can judge if we are doing better or worse. So, we can improve our performance by introducing MDP Approach to help our Instinctive Cognition.

This is the greatest difference between MTS and MDP Approach. MTS is a static pattern identification, But MDP Approach is a management tool to win the game, utlizing our Instinct Judgement. The greatest difference between MTS and MDP Approach is MDP is a tool for Learning from Failures. We try one way and if it does not work, we will try another.

\section{Applications}

As everybody knows, there are no robots which can learn to swim by themselves. There are robots which can swim, but if the current changes, they cannot adapt to them. To my knowledge, no robots are developed which can learn to swim and which can swim against the flow to get to the goal, no matter how the flow might change. MDP Approach provides us with a support to win such a game.

Therefore, we can develop Wearable Robots, which can learn how to win the game by trial and error. So, if we put Wearable Robots on and work together, we can learn to swim our own way. As our body builds are different, how we learn to swim or move varies from person to person, or from situation to situation. And such Wearable Robots will assist seniors to move their own way. And they will facilitate Rehabiliation Customization. Current rehabilitation is trying to get the handicapped back to the nonhandicapped day conditions. For example, if we put on prostheses, then it is a combination of artificial thing and our natural thing (body). So, the movement should be different. We should develop walking or grasping style for the handicapped to enjoy their own way.

\section{Direct Interaction with the Real World: Learning from the Octopus}

The real world is changing frequently, extensively and in an unpredicable manner. So, we need to directly interact with the real world. In addition, materials are gettting softer and softer, so that vision alone cannot identify the object. We need to directly interact with the object. That is why haptics or sense of touching is getting importance.

This is what the octopuses are doing. They die immendiately after their babies are born. So, they do not inherit knowledge from the previous generations. They live on their instinct alone. But they are known as the expert of escape and they can escape from any environments and situations. They directly interact with the real world. That is why they can. MDP Approach helps us to make the most of our instincts to perceive and make appropriate decisions.

\section{Summary}

As Abraham Maslow pointed out in his hierarchy of human needs [7], the highest need of us, humans, is Self-Actualization. And Edward Deci and Richard Ryan proposed 
Self-Determination Theory and they pointed out if the work is intrinsically motivated and self-determined, we will have the greatest mental satisfaction and happiness. They also pointed out that growth is important human need [8]. But they did not tell us how. Therefore, Mahalanobis Distance-Pattern Approach is proposed for holistic and qunatitative performance indicator to help us evaluate our performance.

what is unique with MDP Approach is the fiollowing.

To avoid computation complexity, it depends on our Instinct, just the same way as the octopus interacts with the real world directly and make decisions based on their Instinct.

Jean Piaget pointed out in his Theory of Cognitive Development that babies directly interact with the real world and they learn to grow. Until two years old, they move around and interact directly with the real world to learn and grow[9].

MDP Approach may be described as a tool for grownups to learn the same way as babies do or as the octopuses do. It should also be added that even when we say "I can swim". It means different things for different people. Some just float in the water, Others swim beautifully ahead. But both styles are "I can swim". Thus, up to now, we made efforts to be objective, but our styles of movement and decsion making vary widely from person to person. Our body builds and Instinct are different from person to person. Thus, MDP Approach is developed to be subjective for us to enjoy our Self.

Therefore, Emotional Engineering plays an important role for motivating us to challenge such issues and for bringing us with psychological satisfaction and happiness [10],[11],[12].

\section{References}

[1] https://en.wikipedia.org/wiki/Nikolai Bernstein

[2] N.A. Bernstein, The co-ordination and regulation of movements, Pergamon Press, Oxford, 1967.

[3] https://en.wikipedia.org/wiki/Mahalanobis_distance

[4] V. Kostov, S. Fukuda, M. Johansson, Method for Simple Extraction of Paralinguistic Features in Human Face, Image \& Visual Computing, The Journal of the Institute of Image Electronics Engineers of Japan, Vol.30, No.2, pp. 111-125, 2001.

[5] V. Kostov, H. Yanagisawa, M. Johansson, S. Fukuda, Method for Face-Retrieval Using a Cartoon Emotional Expression Approach, JSME Int. Journal, Mechanical Systems, Machine Elements and Manufacturing, Vol.44, No.2, pp.515-526, 2001.

[6] G. Taguchi, S. Chowdhury, Y. Wu, The Mahalanobis-Taguchi System, McGraw-Hill Professional, New York, 2000.

[7] A. H. Maslow, A Theory of Human Motivation, Psychological Review, Vol.50, No.4, 1943.

[8] E. Beci \& R. M. Ryan, Intrinsic Motivation and Self-Determination in Human Behavior, Plenum Press, New York, 1985.

[9[ https://en.wikipedia.org/wiki/Piage's_theory_of_congnitive_development

[10] S. Fukuda (ed.). Emotional Engineering, Springer, London, Vol.1, 2011-Vol.8, 2020.-

[11] S. Fukuda. Self Engineering: Learning from Failures, Springer, London, 2019.

[12] S Fukuda. World 2.0: From Working for Others to Working for Yourself, Sprnger, London, 2020. 